\title{
Left Ventricular Assist Device Implantation via Bi-Thoracotomy Technique: A Single-Center Perspective
}

\author{
Elizabeth Stoeckl ${ }^{1}$, Jason Smith $^{1}$, Ravi Dhingra ${ }^{1}$, and Amy Fiedler ${ }^{1}$ \\ ${ }^{1}$ University of Wisconsin
}

February 24, 2021

\begin{abstract}
Background: Left ventricular assist devices (LVAD) are standardly implanted via full sternotomy. Non-sternotomy approaches are gaining popularity, but potential benefits of this approach have not been well-studied. We hypothesized that LVAD implantation by bi-thoracotomy (BT) would demonstrate smaller and more consistent inflow cannula angles leading to improved postoperative outcomes compared to sternotomy. Methods: Charts of patients who underwent LVAD implantation between June 2018 and June 2020 at a single academic institution were retrospectively reviewed. Patient demographics, surgical approach (sternotomy vs. BT), laboratory values, and postoperative course were compared. The inflow cannula angle was measured on the first chest radiograph available postoperatively. Results: Of 40 patients studied, BT approach was used in 17 (42.5\%). Mean inflow cannula angles were smaller in BT patients (23.0 vs. 37.1 degrees, $\mathrm{p}=0.018$ ) and had a smaller standard deviation (13.8 vs. 20.3). Excluding patients who went on to receive heart transplant or died in the same hospitalization, there was no difference in median length of hospital stay after surgery (16.0 vs. 17.5 days, $\mathrm{p}=0.768$ ). However, BT patients required fewer days of postoperative inotrope support ( 4.0 vs. 7.0 days, $\mathrm{p}=0.012$ ). Conclusions: Our data suggest inflow cannula angles are smaller and more consistent with the BT approach, which leads to shorter duration of postoperative inotropic support. This finding may suggest improved right heart function following LVAD implant via BT approach. Further study is warranted to determine additional benefits of the BT approach.
\end{abstract}

\section{Introduction}

Left ventricular assist device (LVAD) therapy confers both a survival and quality of life benefit for advanced heart failure patients. ${ }^{1}$ LVADs have standardly been implanted via full median sternotomy, ${ }^{2}$ but the smaller size of newer centrifugal pumps has facilitated the use of less invasive, sternal-sparing techniques, either via bilateral thoracotomies or upper hemi-sternotomy with left thoracotomy. ${ }^{3-5}$ Non-sternotomy LVAD implantation techniques have been shown to be safe and effective when both Heartware ${ }^{\mathrm{TM}} \mathrm{HVAD}^{\mathrm{TM}}$ (Medtronic, Minneapolis, MN) ${ }^{6,7}$ and Heartmate $3^{\mathrm{TM}}$ (HM3) (Abbott, Chicago, IL) ${ }^{8,9}$ devices are used, and they have demonstrated lower rates of right ventricular failure, blood product transfusions, and lengths of hospital stay by comparison.

Several advantages of sternal-sparing approaches compared to sternotomy have been proposed. There may be less risk of right heart failure, a common complication after LVAD implantation, ${ }^{10}$ because the pericardium over the right ventricle is preserved. ${ }^{8}$ With the heart maintained in anatomic position, there is less potential for pulmonary artery obstruction and coronary hypoperfusion. ${ }^{8}$ Additionally, better visualization of the apical inflow anastomosis and assessment of hemostasis are afforded, and the sternum is spared for more easy sternal entry if the patient is to move forward with heart transplantation following LVAD implant. Finally, without sternal restrictions, patients can begin more prompt physical therapy after surgery, which is beneficial for rehabilitation in both bridge-to-transplant and destination therapy patients. ${ }^{8}$

In addition to improvements in newer LVAD generations and development of new implantation techniques, in- 
flow cannula angulation has also been tied to patient outcomes. In HVAD patients, Imamura et al.associated smaller cannula coronal angles with improved left ventricular unloading, ${ }^{11}$ leading to improved myocardial structural and functional parameters that help prevent heart failure recurrence. ${ }^{12}$ Furthermore, angles [?] $65^{\circ}$ were associated with reduced heart failure readmission rates. ${ }^{11}$ Prior work has also suggested that inflow cannula angles after LVAD implantation can be more consistently reproduced with a sternal-sparing approach, compared to median sternotomy. ${ }^{13}$

Increasing adoption of sternal-sparing approaches to LVAD implantation underscores the need for individual institutions to illustrate their experience and patient outcomes using these techniques. The purpose of this study is to compare both inflow cannula angles and postoperative outcomes for patients who received LVAD implantation by either median sternotomy or bi-thoracotomy (BT).

\section{Patients and Methods}

Study Design and Patient Characteristics

This study was approved by the University of Wisconsin-Madison Institutional Review Board. A retrospective review was performed of all patients who received LVAD implantation, with either HM3 LVAD or primarily with the HeartWare HVAD System, in the Cardiothoracic Surgery division at the University of Wisconsin between June 2018 to June 2020. Patients undergoing an operation for LVAD pump exchange were excluded from the study. Medical records were reviewed for patient demographics, cardiac surgery history, surgical approach to LVAD implantation (sternotomy vs. BT), past medical history, preoperative right heart catheterization, preoperative ejection fraction, INTERMACS profile, need for preoperative hemodynamic support, and postoperative hospital course.

The inflow cannula angle was measured by one reviewer (EMS) on the first chest radiograph available postoperatively using the angle measurement tool within the Change Healthcare Radiology Solutions ${ }^{\text {TM }}$ PACS system. The angle was recorded as the line above the horizontal plane, and the horizontal plane was measured from a line visually fitted to the vertebral bodies (Figure 1). The measurements were validated by a board-certified chest radiologist (ESK).

Operative Approach

Patients were selected for LVAD implantation as either destination therapy or bridge to transplantation by our multidisciplinary team. Three different cardiac surgeons (TK, AGF, JWS) performed LVAD implantation for this patient population during the study period. It is beyond the scope of this manuscript to describe in depth technical details of each approach.

Prior to May 2019, the BT approach was not utilized at our institution and all LVADs were implanted via full median sternotomy. The BT approach became our institutional standard in October 2019 for all LVAD implants.

Statistical Analysis

Data are presented as mean \pm standard deviation for normally distributed variables and median (interquartile range) for non-normally distributed variables. The Independent-Samples t-test was used for normally distributed continuous variables and the Mann-Whitney U-test was used for non-normally distributed continuous variables. The $\mathrm{X}^{2}$ or the Fisher's Exact test were used for categorical variables. All tests were two-sided, and the level of statistical significance was set at $\mathrm{p}<0.05$. All analyses were performed using IBM SPSS®) version 27 (IBM Corporation, Armonk, NY).

\section{Results}

Between June 2018 and June 2020, 44 patients underwent LVAD implantation. Four of these patients were undergoing device exchange and were excluded. Of the 40 included, 17 (42.5\%) devices were placed by BT approach and $23(57.5 \%)$ by sternotomy. The BT and sternotomy groups did not have significant differences in median age at the time of operation (62.0 vs. 55.0 years, $\mathrm{p}=0.274)$, sex (14 vs. 19 males, $\mathrm{p}>0.99)$, or mean 
BMI (27.4 vs. $30.0, \mathrm{p}=0.170$ ) (Table 1). Likewise, the groups did not demonstrate significant differences in preoperative comorbidities or laboratory values, except for cardiac index ( 2.2 vs. 1.8 liters $/ \mathrm{min} / \mathrm{m} 2, \mathrm{p}=0.020)$ and serum sodium (137.7 vs. $134.7 \mathrm{mEq} / \mathrm{L}, \mathrm{p}=0.006$ ) (Table 1 ). There was no difference in the most recent ejection fraction prior to surgery ( 15.0 vs. $20.0 \%, \mathrm{p}=0.460$ ) or in the Interagency Registry for Mechanically Assisted Circulatory Support (INTERMACS) profile (3.0 vs. 3.0, p=0.341) (Table 1). Furthermore, there was no difference in the percentage of patients who required preoperative hemodynamic support in the form of inotropes, intra-aortic balloon pump (IABP), or extracorporeal membrane oxygenation (ECMO), with $76.5 \%$ of BT and $82.6 \%$ of sternotomy patients requiring one or more of these modalities $(\mathrm{p}=0.702)$ (Table 1). Lastly, more BT patients were planned as destination therapy (DT) than sternotomy patients ( $88.2 \%$ vs. $52.2 \%, \mathrm{p}=0.020)$ (Table 1).

In comparing postoperative outcomes, BT patients demonstrated a lower median intraoperative blood loss than sternotomy patients $(0.0$ vs. $100.0 \mathrm{~mL})$, though these values did not reach statistical significance $(\mathrm{p}=0.096)$ (Table 2). Excluding patients who went on to receive a heart transplant $(\mathrm{n}=8)$ or died $(\mathrm{n}=14)$, there was no difference in median length of hospital stay after surgery (16.0 vs. 17.5 days, $\mathrm{p}=0.768)$. BT patients required fewer days of postoperative inotrope support ( 4.0 vs. 7.0 days, $\mathrm{p}=0.012$ ). Additionally, the mean inflow cannula angles measured on the first postoperative chest radiograph were smaller in BT patients (23.0 vs. 37.1 degrees, $\mathrm{p}=0.018$ ) and had a smaller standard deviation (13.8 vs. 20.3).

\section{Comment}

In this study, we demonstrate notable postoperative differences between patients receiving LVAD implantation by BT and sternotomy techniques. We add our institution's experience to the growing body of evidence supporting sternal-sparing techniques as safe and effective alternatives to median sternotomy. ${ }^{6-9}$ Moreover, in our cohort of patients, inflow cannula angles were smaller and more consistent when the BT technique was used. We therefore validate the findings of Ayerset al., who also demonstrated smaller and more consistent inflow cannula angles after sternal-sparing implantation in patients who received the HM3 LVAD. ${ }^{13}$

We found that BT patients required fewer days of postoperative inotrope support compared to sternotomy patients, similar to early clinical outcomes reported by Wood et al. ${ }^{8}$ The Interagency Registry for Mechanically Assisted Circulatory Support (INTERMACS) considers duration of post-implant inotropes when defining the severity of right ventricular failure, ${ }^{10}$ and other work has found significantly lower incidence of severe right ventricular failure in sternal-sparing implants. ${ }^{9}$ Thus, our findings may be suggestive of less right heart failure in patients who received their implant by BT, though further prospective work will be needed to evaluate post-operative right heart function in the index hospitalization and ultimately long-term outcomes following discharge. Intraoperative blood loss and shorter length of index hospitalization both favored the BT cohort but did not reach statistical significance. This could be due to our relatively small sample size, since shorter hospitalizations after sternal-sparing implants have been demonstrated in prior work, ${ }^{6-9}$ and in general, minimally invasive cardiac surgery techniques are associated with less blood loss. ${ }^{14,15}$

Regarding inflow cannula angulation, Imamura et al. associated smaller cannula coronal angles with left ventricle unloading (smaller left ventricular diastolic dimension and lower pulmonary capillary wedge pressure) and with reduced heart failure readmission rates. ${ }^{11}$ They offer angles [?]65deg as an appropriate target for HVAD deices to allow consistent blood removal from the left ventricle; steeper angles may introduce turbulent flow which may impede reverse left ventricular remodeling. Sternal-sparing LVAD implantation techniques facilitate optimal cannula angulation by allowing direct visualization of the cardiac apex in anatomic position. ${ }^{7}$ In this way, the other proposed benefits of sternal-sparing techniques, such as preservation of the pericardium surrounding the right ventricle and lack of postoperative sternal restrictions, may be further augmented by improved cannulation. While we do demonstrate smaller and more consistent inflow cannula angles in our BT patients, further work is needed to clarify the impact of cannula angle on patient outcomes, specifically related to right ventricular dysfunction in the postoperative period.

More BT patients in our cohort were planned as destination therapy than sternotomy patients. This is an interesting finding, however, may be a result of timing bias. Since mid-2019, all LVADs have been implanted 
by BT as our standard institutional protocol. This timing aligns with the introduction of the new United Network for Organ Sharing (UNOS) allocation system for heart transplantation, which since implementation, has shifted transplant prioritization away from patients on the waiting list living with a durable LVAD to those who are more acutely ill on temporary circulatory support. ${ }^{16}$

Re-operative median sternotomy is a known risk factor for postoperative morbidity and mortality in patients who are bridged to transplant; however, patients whose sternotomy was for LVAD placement have shown equivalent survival to primary cardiac transplant patients, suggesting that the medical stabilization conferred by LVAD therapy may mitigate the risks posed by re-operative sternotomy. ${ }^{17}$ While LVAD placement by sternotomy may not adversely affect survival after transplant, subjectively, our experience is that sternalsparing approaches to LVAD placement do make subsequent transplant easier, with anecdotally less bleeding and less time on cardiopulmonary bypass due to ease of dissection.

Our work has several limitations inherent to its design as a retrospective, single-institution study, which may limit its generalizability. HVAD is the most often used device at our institution; however, HM3 is also utilized at our institution and across the country. As we gain comfort with non-sternotomy approaches for LVAD implantation, future investigation is warranted to confirm findings across all pump models and non-sternotomy approaches.

In conclusion, our data suggest inflow cannula angles are smaller and more consistent with the BT approach, which has been associated with improved patient outcomes. Patients who underwent BT required fewer days of postoperative inotrope support. Further research is needed to quantify the clinical impact of the BT approach to LVAD implantation more fully, specifically as it relates to right heart dysfunction following LVAD implantation both in the short and long term.

\section{Acknowledgements:}

We thank Eva Kusmirek, MD for her assistance in validating the radiologic findings of this study.

\section{Disclosures:}

Jason Smith is a consultant for Medtronic. Other authors have no financial disclosures.

\section{References}

1. Rose EA, Gelijns AC, Moskowitz AJ, Heitjan DF, Stevenson LW, Dembitsky W, et al. Long-Term Use of a Left Ventricular Assist Device for End-Stage Heart Failure. N Engl J Med 2001;345:1435-43. https://doi.org/10.1056/NEJMoa012175.

2. Slaughter MS. Implantation of the HeartWare Left Ventricular Assist Device. Semin Thorac Cardiovasc Surg 2011;23:245-7. https://doi.org/10.1053/j.semtcvs.2011.07.008.

3. Schmitto JD, Molitoris U, Haverich A, Strueber M. Implantation of a centrifugal pump as a left ventricular assist device through a novel, minimized approach: Upper hemisternotomy combined with anterolateral thoracotomy. J Thorac Cardiovasc Surg 2012;143:511-3. https://doi.org/10.1016/j.jtcvs.2011.07.046.

4. Popov AF, Hosseini MT, Zych B, Simon AR, Bahrami T. HeartWare Left Ventricular Assist Device Implantation Through Bilateral Anterior Thoracotomy. Ann Thorac Surg 2012;93:674-6. https://doi.org/10.1016/j.athoracsur.2011.09.055.

5. Maltais S, Danter MR, Haglund NA, Schmitto JD, Stulak JM. Nonsternotomy Approaches for Left Ventricular Assist Device Placement. Oper Tech Thorac Cardiovasc Surg 2014;19:276-91. https://doi.org/10.1053/j.optechstcvs.2014.10.001.

6. Maltais S, Anwer LA, Tchantchaleishvili V, Haglund NA, Dunlay SM, Aaronson KD, et al. Left Lateral Thoracotomy for Centrifugal Continuous-Flow Left Ventricular Assist Device Placement. ASAIO J 2018;64:715-20. https://doi.org/10.1097/MAT.0000000000000714. 
7. McGee E, Danter M, Strueber M, Mahr C, Mokadam NA, Wieselthaler G, et al. Evaluation of a lateral thoracotomy implant approach for a centrifugal-flow left ventricular assist device: The LATERAL clinical trial. J Hear Lung Transplant 2019;38:344-51. https://doi.org/10.1016/j.healun.2019.02.002.

8. Wood KL, Ayers BC, Sagebin F, Vidula H, Thomas S, Alexis JD, et al. Complete Sternal-Sparing HeartMate 3 Implantation: A Case Series of 10 Consecutive Patients. Ann Thorac Surg 2019;107:1160-5. https://doi.org/10.1016/j.athoracsur.2018.10.005.

9. Gosev I, Wood K, Ayers B, Barrus B, Knight P, Alexis JD, et al. Implantation of a fully magnetically levitated left ventricular assist device using a sternal-sparing surgical technique. J Hear Lung Transplant 2020;39:37-44. https://doi.org/10.1016/j.healun.2019.09.012.

10. Lampert BC, Teuteberg JJ. Right ventricular failure after left ventricular assist devices. J Hear Lung Transplant 2015;34:1123-30. https://doi.org/10.1016/j.healun.2015.06.015.

11. Imamura T, Adatya S, Chung B, Nguyen A, Rodgers D, Sayer G, et al. Cannula and Pump Positions Are Associated With Left Ventricular Unloading and Clinical Outcome in Patients With HeartWare Left Ventricular Assist Device. J Card Fail 2018;24:159-66. https://doi.org/10.1016/j.cardfail.2017.09.013.

12. Al-Sarie M, Rauf A, Kfoury AG, Catino A, Wever-Pinzon J, Bonios M, et al. Myocardial Structural and Functional Response After Long-Term Mechanical Unloading With Continuous Flow Left Ventricular Assist Device. JACC Hear Fail 2016;4:570-6. https://doi.org/10.1016/j.jchf.2016.02.015.

13. Ayers B, Marini T, Wood K, Kim S, Croake M, Bernstein W, et al. Sternal-Sparing Approach for LVAD Implantation Allows for a More Consistent Inflow Cannula Angle. J Hear Lung Transplant 2020;39:S445. https://doi.org/10.1016/j.healun.2020.01.261.

14. Cohn LH, Adams DH, Couper GS, Bichell DP, Rosborough DM, Sears SP, et al. Minimally Invasive Cardiac Valve Surgery Improves Patient Satisfaction While Reducing Costs of Cardiac Valve Replacement and Repair. Ann Surg 1997;226:421-8. https://doi.org/10.1097/00000658-199710000-00003.

15. Haberl T, Riebandt J, Mahr S, Laufer G, Rajek A, Schima H, et al. Viennese approach to minimize the invasiveness of ventricular assist device implantation. Eur J Cardio-Thoracic Surg 2014;46:991-6. https://doi.org/10.1093/ejcts/ezu051.

16. Teuteberg JJ, Cleveland JC, Cowger J, Higgins RS, Goldstein DJ, Keebler M, et al. The Society of Thoracic Surgeons Intermacs 2019 Annual Report: The Changing Landscape of Devices and Indications. Ann Thorac Surg 2020;109:649-60. https://doi.org/10.1016/j.athoracsur.2019.12.005.

17. Axtell AL, Fiedler AG, Lewis G, Melnitchouk S, Tolis G, D'Alessandro DA, et al. Reoperative sternotomy is associated with increased early mortality after cardiac transplantation. Eur J Cardio-Thoracic Surg 2019;55:1136-43. https://doi.org/10.1093/ejcts/ezy443.

Tables

Table 1. Patient Demographics and Clinical Characteristics by Operative Approach for LVAD Implantation

\begin{tabular}{llll}
\hline Variable & Bi-Thoracotomy $(\mathbf{n = 1 7})$ & Sternotomy $(\mathbf{n = 2 3})$ & $\mathbf{P}$-value \\
\hline Age at operation, years (IQR) & $62.0(55-66)$ & $55.0(41-66)$ & 0.274 \\
Male (\%) & $14(82.4)$ & $19(82.6)$ & $\mathrm{P}>0.99$ \\
BMI, $\mathrm{kg} / \mathrm{m}^{2}$ & $27.4 \pm 5.3$ & $30.0 \pm 6.4$ & 0.170 \\
INTERMACS profile, median (IQR) & $3.0(2-4)$ & $3.0(2-3)$ & 0.341 \\
Profile 1 & 3 & 2 & \\
Profile 2 & 2 & 5 & \\
Profile 3 & 4 & 9 & \\
Profile 4 & 5 & 2 & \\
Profile 5 & 0 & 0 &
\end{tabular}




\begin{tabular}{llll}
\hline Variable & Bi-Thoracotomy $(\mathbf{n = 1 7})$ & Sternotomy $(\mathbf{n = 2 3 )}$ & P-value \\
\hline Profile 6 & 1 & 0 & \\
Comorbidities & $5(29.4)$ & $6(26.1)$ & $\mathrm{P}>0.99$ \\
Previous cardiac surgery (\%) & $15(88.2)$ & $18(78.3)$ & 0.677 \\
Arrhythmia History (\%) & $8(47.1)$ & $10(43.5)$ & $\mathrm{P}>0.99$ \\
Diabetes (\%) & $3(17.6)$ & $2(8.7)$ & 0.634 \\
Stroke or TIA (\%) & $15(88.2)$ & $18(78.3)$ & 0.677 \\
CKD (\%) & 2 & 1 & \\
with dialysis & $4(23.5)$ & $3(13.0)$ & 0.432 \\
COPD (\%) & & & \\
Pre-operative laboratory values & $15.0(10-20)$ & $20.0(15-20)$ & 0.460 \\
LVEF (IQR) & $2.2 \pm 0.5$ & $1.8 \pm 0.5$ & $\mathbf{0 . 0 2 0}$ \\
Cardiac Index, liters/min/m ${ }^{2}$ & $23.4 \pm 8.8$ & $25.5 \pm 9.3$ & 0.476 \\
PCWP, mm Hg & $35.3 \pm 12.9$ & $13.6 \pm 10.0$ & 0.934 \\
Mean PAP, mm Hg & $10.7 \pm 5.6$ & $242.8 \pm 115.8$ & 0.174 \\
RAP, mm Hg & $243.4 \pm 158.8$ & $54.8 \pm 9.8$ & 0.989 \\
PVR Preop, dynes·sec·cm ${ }^{-5}$ & $57.5 \pm 11.5$ & $134.7 \pm 2.7$ & 0.455 \\
SvO2 (\%) & $137.7 \pm 3.6$ & $31.0(23-48.5)$ & $\mathbf{0 . 0 0 6}$ \\
Sodium & $28.0(20-37)$ & $1.5 \pm 0.5$ & 0.179 \\
BUN (IQR) & $1.3 \pm 0.5$ & $28.0(19-39)$ & 0.379 \\
Cr & $23.0(19-33)$ & $32.0(19.5-43.5)$ & 0.631 \\
AST (IQR) & $19.0(17-32)$ & $3.4 \pm 0.5$ & 0.318 \\
ALT (IQR) & $3.2 \pm 0.6$ & $1.1(0.8-1.8)$ & 0.256 \\
Albumin & $0.9(0.5-1.5)$ & $19(82.6)$ & 0.233 \\
Total Bilirubin (IQR) & $13(76.5)$ & 19 & 0.702 \\
Pre-operative support & 12 & 8 & \\
Inotropes & 5 & 0 & \\
IABP & 2 & $11(47.8)$ & \\
ECMO & & $12(52.2)$ & $\mathbf{0 . 0 2 0}$ \\
Intended goal & $2(11.8)$ & & \\
Bridge to Transplant (\%) & $15(88.2)$ & & \\
Destination Therapy (\%) & & & \\
\hline
\end{tabular}

Abbreviations: ALT, alanine transaminase; AST, aspartate transaminase; BMI, body mass index; BUN, blood urea nitrogen; CKD, chronic kidney disease; COPD, chronic obstructive pulmonary disease; ECMO, extracorporeal membrane oxygenation; IABP, intra-aortic balloon pump; INTERMACS, Interagency Registry for Mechanically Assisted Circulatory Support; LVEF, left ventricular ejection fraction; PAP, pulmonary artery pressure; PCWP, pulmonary capillary wedge pressure; RAP, right atrial pressure; SvO2, mixed venous oxygen saturation; TIA, transient ischemic attack

${ }^{a}$ Variables are expressed as number $(\%)$ and mean \pm standard deviation if normally distributed or median (interquartile range) if non-normally distributed.

${ }^{\mathrm{b}} \mathrm{P}$-values are bolded to indicate statistical significance.

Table 2. Outcomes After LVAD Implantation by Operative Approach

\begin{tabular}{llll}
\hline Variable & Bi-Thoracotomy $(\mathbf{n}=\mathbf{1 7})$ & Sternotomy $(\mathbf{n}=\mathbf{2 3})$ & $\mathbf{p}$-value \\
\hline Intraoperative blood loss, mL (IQR) & $0.0(0-250)$ & $100.0(50-400)$ & 0.096 \\
Days of postoperative inotrope support (IQR) & $4.0(2-5)$ & $7.0(4-12)$ & $\mathbf{0 . 0 1 2}$ \\
Days of postoperative hospitalization (IQR) & $16.0(13-23)$ & $17.5(12.25-30.75)$ & 0.768 \\
\hline
\end{tabular}




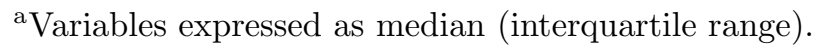

${ }^{\mathrm{b}} \mathrm{P}$-values are bolded to indicate statistical significance.

\section{Figure Legend}

Sample Inflow Cannula Angle Measurement on Postoperative Chest Radiograph

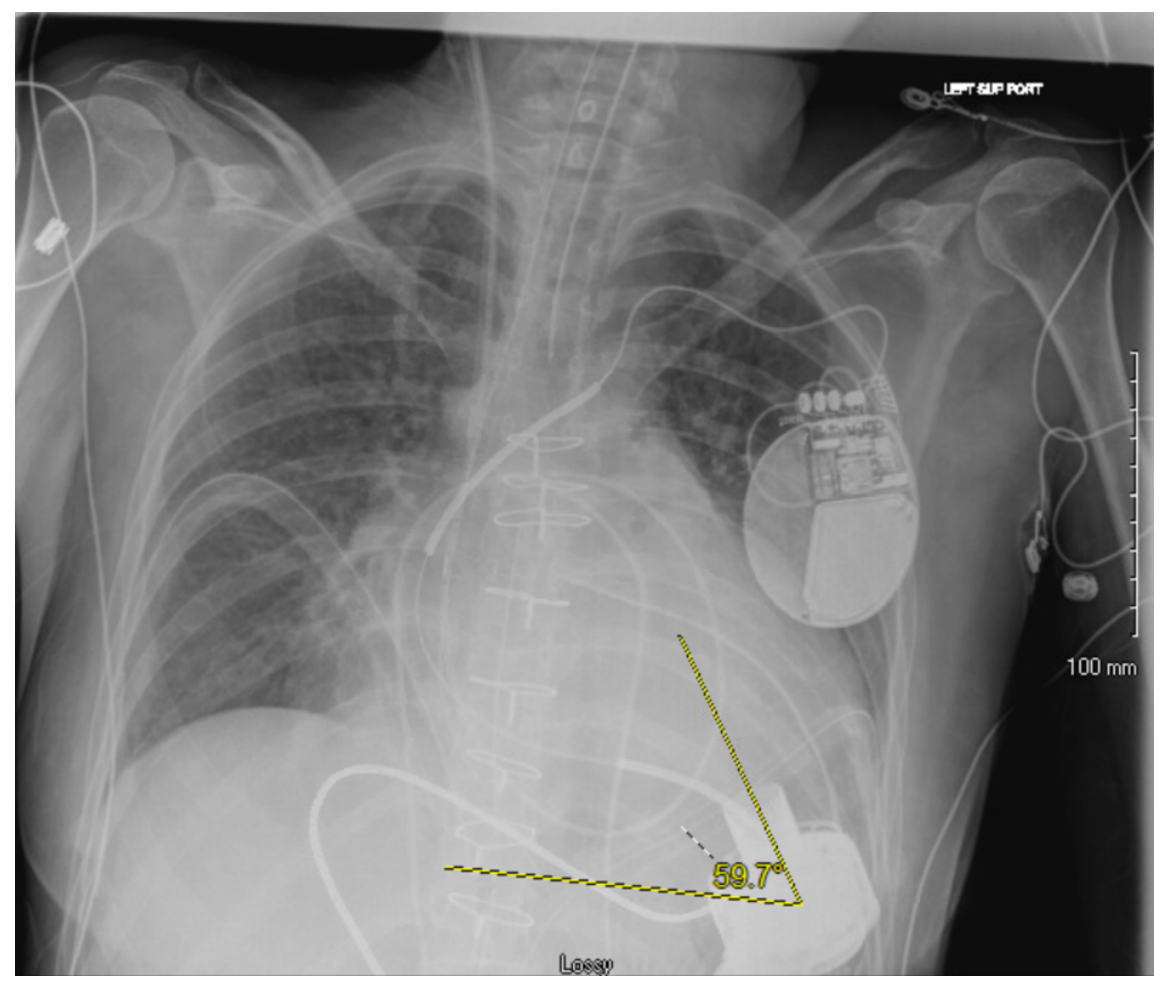

